\title{
Dietary fat intake and blood pressure in UK adolescents: a longitudinal study
}

\author{
Ziyi Li, Charlotte E.L Evans and Janet E. Cade \\ Nutritional Epidemiology Group, School of Food Science and Nutrition, University of Leeds, LS2 9JT, Leeds, UK.
}

Studies suggest that higher dietary fat intake is associated with higher blood pressure levels ${ }^{(1,2)}$, but relevant studies in adolescents, especially longitudinal studies are lacking. The aim of this study is to determine the longitudinal relationship between dietary fat intake and blood pressure in UK adolescents.

A sample of 6282 participants was selected from the Avon Longitudinal Study of Parents and Children (ALSPAC). General information and blood pressure data were collected at age 10,11,12,13,15 and 17 years. Diet was assessed using a four-day food record at age 10 and 13 years. BP data was transformed to systolic/diastolic BP Z-scores (SBPZ/DBPZ). Multivariable linear regression was undertaken to explore the relationships between fat intake and future BPZ. Participants were grouped into low/medium/high fat intake group by their total fat intake tertiles. Incidence rate and mean survival time was calculated in each group. Cox regression was used to explore the longitudinal relationship between fat intake and the risk of hypertension. Gender, ethnicity, BMI, alcohol intake, smoking status, sodium intake, energy intake excluding fat, parents hypertension history and household income were adjusted for in each model.

In fully adjusted linear models, each $1 \mathrm{mg}$ increase in fat intake at age 10 was associated with 0.017 (95\% CI: 0.001 to 0.034) higher DBP at age 17 years. Each $1 \mathrm{mg}$ increase in fat intake at age 13 years was associated with 0.026 (95\% CI: 0.06 to 0.48$)$ higher SBP at age 15 years. The incidence rate in the low fat group $(5.5 \%)$ was lower than medium $(6 \cdot 2 \%) /$ high intake $(6 \cdot 1 \%)$ group, and the mean survival time of the low intake group ( 7.3 years) was higher than medium ( $7 \cdot 1$ years) and high intake ( $7 \cdot 2$ years) group. Cumulative hazard estimates in each group can be seen in Figure 1. In cox regression models, no relationship between fat intake and hazard ratio of hypertension was found.
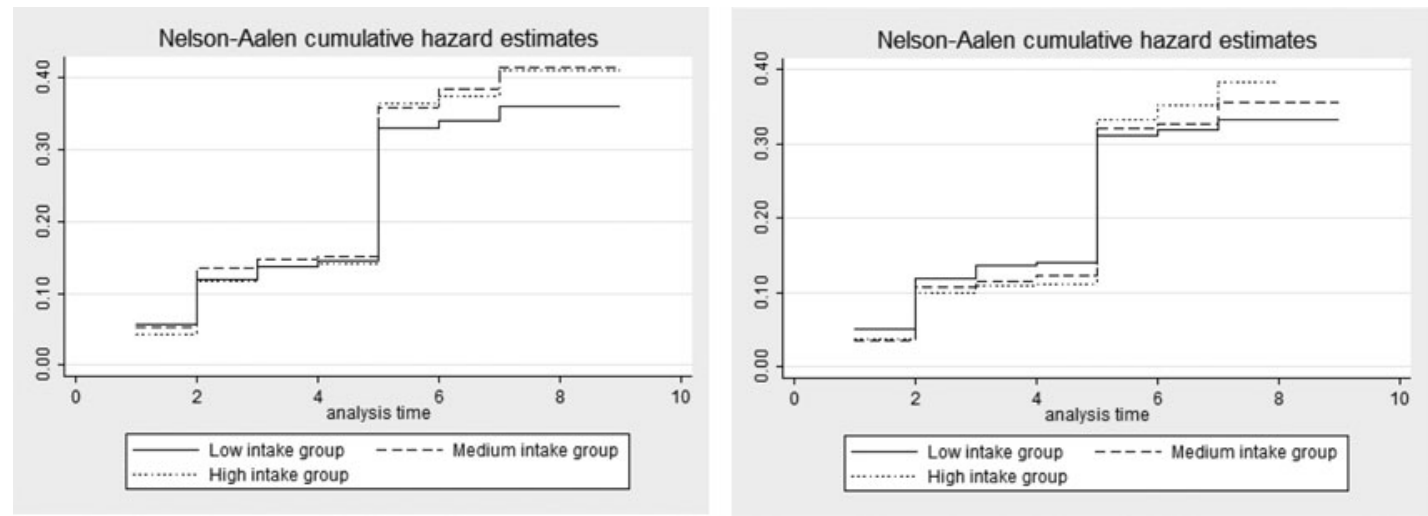

Fig. 1. Cumulative hazard estimates in each fat intake group at 10 years (left) and 13 years (right).

In conclusion, in the ALSPAC population, a positive association was found between dietary fat intake at 10 years and DBP at 17 years, also between fat intake at 13 years and SBP at 15 years. The low fat intake group tended to have lower incidence rate and higher survival time compared to the medium/high intake groups, but no statistically significant association was found in the fully adjusted Cox regression.

1. Aeberli I et al. (2009) Diet determines features of the metabolic syndrome in 6- to 14-year-old children. Int J Vitam Nutr Res 79(1), p. 14-23.

2. Mirza NM et al. (2013) Effects of a low glycemic load or a low-fat dietary intervention on body weight in obese Hispanic American children and adolescents: a randomized controlled trial. Am J Clin Nutr 97(2), p. 276-85. 